\title{
Transverse transport in coupled strongly correlated electronic chains
}

\author{
S. Capponi and D. Poilblanc \\ Laboratoire de Physique Quantique and C.N.R.S. (UMR 5626), \\ Université Paul Sabatier, 31062 Toulouse, France.
}

\begin{abstract}
One-particle interchain hopping in a system of coupled Luttinger liquids is investigated by use of exact diagonalizations techniques. Firstly, the two chain problem of spinless fermions is studied in order to see the behaviour of the band splitting as a function of the exponent $\alpha$ which characterizes the $1 D$ Luttinger liquid. We give numerical evidence that inter-chain coherent hopping (defined by a non-vanishing splitting) can be totally suppressed for $\alpha \sim 0.4$ or even smaller $\alpha$ values. The transverse conductivity is shown to exhibit a strong incoherent part even when coherent inter-chain hopping is believed to occur (at small $\alpha$ values). Implications for the optical experiments in quasi-1D organic or high- $T_{c}$ superconductors is outlined.
\end{abstract}

Recently, the study of strongly correlated fermions confined to coupled chains has received a great deal of interest in particular as a way of studying the dimensional cross-over from 1D Luttinger-like behaviour to 2D.

Some time ago, Anderson emphasized the crucial difference between in-plane and inter-plane (c-axis) transport observed in copper oxide superconductors [2]. Indeed, experimentally the transverse conductivity has a completely incoherent frequency dependence [3]: there seems to be no sizeable Drude-like term (except in the optimally doped systems) and $\sigma(\omega)$ is a very slowly increasing function of the frequency. This phenomenon has been interpreted as an incoherent hopping or as the "confinement" of the electrons inside the weakly coupled planes 顿. However, for coupled Fermi liquids (FL), Landau theory predicts coherent transverse hopping and no anomalous transport. Therefore, these data have suggested that the ground state (GS) of the two-dimensional (2D) plane itself is not of the usual FL type. Unfortunately, it has been impossible yet to prove the NFL nature in $2 \mathrm{D}$ except for unrealistic models.

However, it is well known that the generic features of correlated 1D electrons are not FL-like but rather those of a Luttinger liquid (LL) [5] and the precise nature (asymptotic behaviour, exponents, etc...) of the system can be easily controlled. In addition, quasi-1D systems are realized in nature, and the problem of coupled chains is of direct relevance there. For instance, in the case of the organic superconductors of the (TMTSF) $)_{2} \mathrm{X}$ family [6], also known as the Bechgaard salts, the high temperature properties are believed to be essentially one-dimensional, while the low-temperature behaviour is rather two- or three-dimensional. This cross-over is presumably responsible for the anomalies observed in the temperature dependence of several quantities (such as the $2 k_{F}$ contribution to the relaxation rate [7], the ratio of the perpendicular conductivity to the parallel one [6], the plasma edge when the electric field is polarized perpendicular to the chains $[6], \ldots)$ as well as for the insulating behaviour reported for (TMTSF) ${ }_{2} \mathrm{ClO}_{4}$ in the presence of a strong enough magnetic field [8]. Theoretically a lot of work has already been devoted to that problem but several as- pects of this cross-over have to be understood better, in particular those concerned with the transport properties perpendicular to the chains.

Hence, from now on and for sake of simplicity, we shall only deal with weakly coupled chains.

The effect of single-particle transverse hopping has previously been studied from a renormalization group point of view [9]. Let us recall here that a LL has a different structure from FL: there are no quasi-particle like excitations but instead collective modes (charge and spin) with different velocities which lead to the so-called spincharge separation; moreover, the density of states $n(k)$ has no step-like structure at the Fermi level but instead a power law singularity $n(k)-n\left(k_{F}\right) \sim\left|k-k_{F}\right|^{\alpha}$ defining the parameter $\alpha$ which depends on the intra-chain interaction. It turns out that the hopping $t_{\perp}$ is a relevant perturbation when $\alpha \leq 1$ [9].

However, it has been argued that relevance in that sense was not necessarily a sufficient condition to cause coherent motion between chains. This, e.g., can be seen from the following model [10]; let a system of two separated chains be prepared at time $t=0$ with a difference of $\Delta N$ particles between the two chains. Then, the interchain hopping is turned on and one considers the probability of the system of remaining in its initial state, $P(t)$. Coherence or incoherence can then be defined as the presence or absence of oscillations in $P(t)$.

In Ref. [10], the authors found two regimes for $P(t)$ which depend entirely on the value of $\alpha$ : the case $\alpha<$ $1 / 2$ exhibited coherent motion while $\alpha>1 / 2$ showed no signal of coherence (see also discussion in [1]).

This paper is devoted to the study of various aspects of interchain coherence in systems of strongly correlated spinless fermions. A more extended version of this work can be found in Ref. [1]. We shall derive several quantities sensitive to the coherent/incoherent nature of the hopping transverse to the chains from exact diagonalizations of small systems by the Lanczos algorithm [12].

We consider here a model of spinless fermions on a lattice formed by $m$ chains of length $L$ with a weak interchain hopping: 


$$
\begin{gathered}
H=-\sum_{j, \beta}\left(c_{j+1, \beta}^{\dagger} c_{j, \beta}+\text { H.c. }\right)-t_{\perp} \sum_{j, \beta}\left(c_{j, \beta+1}^{\dagger} c_{j, \beta}+\text { H.c. }\right) \\
+\sum_{j, \beta, \delta} V(\delta) n_{j, \beta} n_{j+\delta, \beta}
\end{gathered}
$$

where $\beta$ labels the chain $(\beta=1, \ldots, m), j$ is a rung index $(j=1, \ldots, L), c_{j, \beta}$ is the fermionic operator, and $V(\delta)$ is a repulsive interaction between two fermions at a distance $\delta$ (the lattice spacing has been set to one). For convenience, we choose a repulsive interaction of the form $V(i)=2 V /(i+1)$ for $i \leq i_{0}$, with, more specifically, $i_{0}=1,2,3$ which corresponds to an interaction extending up to first, second and third nearest neighbours (NN) respectively.

In the $\mathrm{x}$ and $\mathrm{y}$-directions, we shall use arbitrary boundary conditions (BC) by threading the system with a magnetic flux $\Phi_{x}$ and $\Phi_{y}$ respectively (except for $m=1$ or 2 chains where open $\mathrm{BC}$ are used in the y-direction) which is measured in unit of the flux quantum $\Phi_{0}=h c / e$.

The motivation to introduce flux is two-fold. Firstly, as proposed by Kohn 14 transport properties of a correlated system can be directly measured from the response of the system to a twist in the boundary condition. Secondly, our ultimate goal is to extract quantities in the thermodynamic limit from finite size scaling analysis. It turns out that a simple way to improve the accuracy for a fixed system size is to average over the boundary conditions e.g. over $\Phi_{y}$ and/or $\Phi_{x}$ [15,16].

In order to characterize the behaviour of coupled chains, it is required to, first, compute the parameters of a single chain for the same model. Indeed, one important issue is to study whether interchain transport is a universal function of the LL parameters only or whether it depends on the details of the model. In the case of NN interactions, the hamiltonian (known as the $\mathrm{t}-\mathrm{V}$ model) can be mapped onto a spin chain problem by a JordanWigner transformation and this is exactly soluble by the Bethe ansatz; thus, $\alpha$ is known for each filling [5]. However, for extended interactions in space, a numerical investigation is necessary with the help of conformal invariance identities. It turns out that the exponent $\alpha$ can be related to simple physical quantities 17 which can be easily extracted from standard exact diagonalization results using the Lanczos algorithm.

The simplest approach to investigate interchain coherence is to consider two coupled chains, i.e. a $2 \times L$ ladder. We proceed along the lines of Ref. [13]. In the absence of interaction, $t_{\perp}$ leads to bonding and anti-bonding dispersion bands corresponding to transverse momentum $k_{\perp}=0$ or $\pi$ respectively, as seen in Fig. (11). The splitting $2 t_{\perp}$ between these bands can be viewed as the signature of a coherent transverse hopping. These bands correspond to a $\delta$-function singularity in the single particle hole (electron) spectral function for $k<k_{F}\left(k>k_{F}\right)$.

In the case of interacting particles, this $\delta$-function singularity is replaced by a power law singularity and the elementary excitations are collective modes. Here, we address the issue of the influence of the hopping $t_{\perp}$ on this singularity, in particular we investigate whether a splitting occurs.
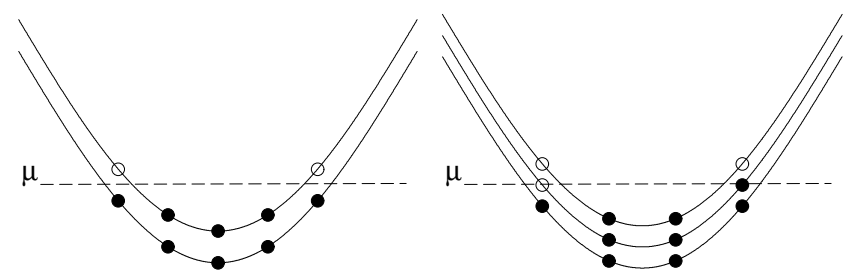

FIG. 1. Dispersion relation along the chain direction in the open shell configurations. Full (open) symbols correspond to occupied (empty) states and $\mu$ is the chemical potential.

As can be seen in Fig.(11), in the open shell configuration, one can add or remove a particle exactly at the Fermi momentum which is independent of the system size. We thus define the splitting as the difference between the electron and hole excitation energies i.e.

$$
\Delta E=E_{0}\left(N_{e}+1, \mathbf{k}_{e}\right)+E_{0}\left(N_{e}-1, \mathbf{k}_{h}\right)-2 E_{0}\left(N_{e}\right) .
$$

where $E_{0}\left(N_{e}\right)$ is the reference energy corresponding to the absolute GS of the $N_{e}=n N$ electron system. The momenta for the electron and hole excitations are fixed, $\mathbf{k}_{e}=\left(k_{F}, \pi\right)$ and $\mathbf{k}_{h}=\left(k_{F}, 0\right)$. For $\mathrm{V}=0$, this expression exactly gives the splitting $2 t_{\perp}$ for any system size. For $t_{\perp}=0$ but finite interaction strength, $\Delta E$ is finite, however it scales to zero in the thermodynamic limit as expected. We observe too that an accurate finite size scaling analysis can be performed for finite interaction strength and finite $t_{\perp}$, assuming $1 / \mathrm{L}$ finite size corrections.

The extrapolated values $(L=\infty)$ of $\Delta E$ are plotted as a function of $t_{\perp}^{1 /(1-\alpha)}$ as suggested by the renormalization group approach [9] for various interactions $\left(i_{0}=2\right.$ model $)$ in Fig. (2).

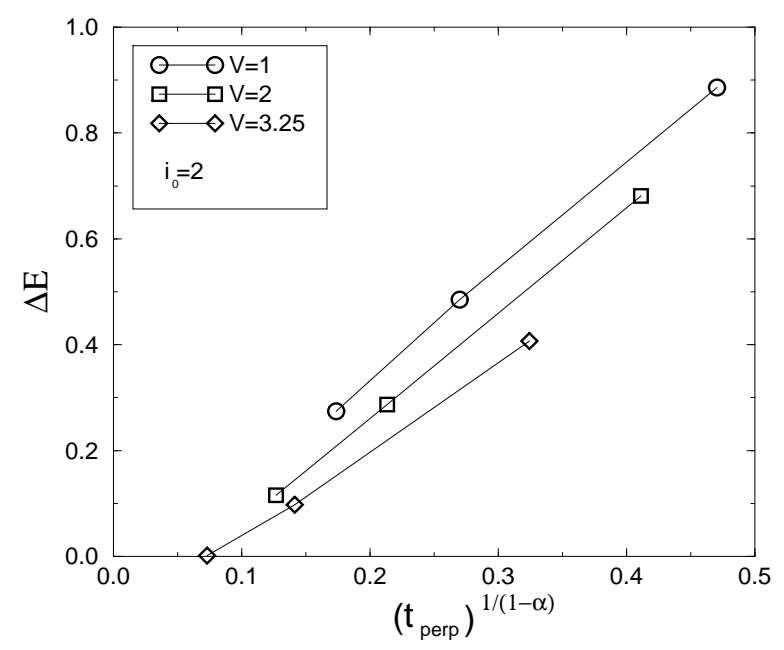

FIG. 2. Extrapolated $(L=\infty)$ values of $\Delta E$ vs $t_{\perp}^{1 /(1-\alpha)}$ in the $i_{0}=2$ spinless fermion model with several values of $V$ (indicated on the figure). 
A strong reduction of this splitting for increasing $\alpha$ indicates that intrachain repulsion has a drastic influence in prohibiting interchain coherent hopping. For large interactions like $V=3.25(\alpha \sim 0.38)$, there is a critical value $t_{\perp}^{*}(\alpha)$ of $t_{\perp}$ below which incoherent transverse hopping takes place. This corresponds to the case $\alpha>\alpha_{0}$. For smaller interaction like $V=1(\alpha \sim 0.08)$ our data are consistent with a coherent behaviour when $t_{\perp} \rightarrow 0$. Hence, transverse hopping remains coherent in this case which corresponds to $\alpha<\alpha_{0}$. For intermediate interactions like $V=2(\alpha \sim 0.22)$, our data are not conclusive. However, very small values of $\alpha_{0}$ like 0.2 (or even smaller) are not inconsistent with our numerical analysis.

When spin is taken into account, the spin-charge separation that occurs in $1 \mathrm{D}$ should suppress even further the coherent transverse hopping. At a qualitative level, this can be understood from the fact that only real electrons can hop from one chain to the next and this is believed to become more difficult in the presence of spin-charge separation [2]. Although dealing with a different filling $n=1 / 3$ and with particles with spin, the same qualitative behaviour is found numerically in Ref. [13]; however, the decrease of the splitting with $\alpha$ is stronger.

The previous study suggests that the interaction tends to confine the electrons within the chains, although no complete confinement seems to occur at small $\alpha$ values where $\Delta E \neq 0$. A better understanding of this phenomenon can be achieved by investigating the transport properties along the y-axis (inter chain) and more precisely the transverse optical conductivity which is the linear response of the system to a spatially uniform, time dependent electric field in the transverse direction. For such a study, a torus geometry is needed $(m \geq 3)$ so that a current can flow around the loop in the y-direction. One of the main advantage of the optical conductivity is that it can directly be measured experimentally.

The real part of the optical conductivity can be written as a sum of two parts, $\sigma_{y y}(\omega)=2 \pi D_{y y} \delta(\omega)+\sigma_{y y}^{r e g}(\omega)$ where $2 \pi D_{y y}$ is the Drude weight in the y-direction.

Note that in order to check the numerical results, we can use an important f-sum rule [19]: the integrated conductivity is proportional to the mean value of the transverse kinetic energy in the ground state.

As originally noted by Kohn [14, $D_{y y}$ can be obtained from the dependence of the ground state energy $E_{0}$ on $\Phi_{y}$ as

$$
2 \pi D_{y y}\left(\Phi_{y}\right)=\frac{m^{2}}{4 \pi} \frac{\partial^{2}\left(E_{0} / N\right)}{\partial \Phi_{y}^{2}}
$$

where $N=m L$ is the number of sites.

The previous quantities have been calculated numerically on finite $3 \times L$ lattices using the Lanczos algorithm. We have chosen a quarter filled band so that the extrapolation of results for $L=4,8$ and 12 is possible.

By choosing adequate boundary conditions along $\mathrm{x}$ open shells can be realized as seen in Fig. (11). In this case, even for a finite system, the Drude weight and the total kinetic energy remains finite down to vanishing $t_{\perp}$ as in the non-interacting case. We first consider a fixed value of the flux $\Phi_{y}$ such that $\frac{\partial E_{0}}{\partial \Phi_{y}}=0$. In that case, finite size effects are found to be already weak for the two largest cases $L=8$ and $L=12$ and the Drude weight is strongly suppressed compared to the $V=0$ case [20].

In order to mimic the case of many parallel chains, an average over $\Phi_{y}$ is realized [15.16] i.e. we calculate $\left\langle D_{y y}\right\rangle_{\Phi_{y}}$ and $I_{y y}=\left\langle\int_{0}^{\infty} \sigma_{y y}(\omega) d \omega\right\rangle_{\Phi_{y}}$ which is obtained by averaging the transverse kinetic energy. We observe that the values obtained for the Drude weight by averaging the $3 \times 8$ data over $\Phi_{y}$ are very close to the ones obtained on the $3 \times 12$ cluster at constant flux. Note that, however, if the number of coupled chains is kept fixed (here $m=3$ ), even in the limit $L \rightarrow \infty$, we expect $\left\langle D_{y y}\right\rangle_{\Phi_{y}} \neq D_{y y}\left(\Phi_{y}\right)$. In any case, when confinement within each of the individual chains starts to occur we do not expect crucial differences between the cases of 3 or of an infinite number of coupled chains (if only the $t_{\perp}$ term couples the chains). In Fig. 3, we plot the Drude weight and the total sum rule as a function of $t_{\perp}$ for a moderate interaction. The behaviour of these two quantities is not incompatible with the $t_{\perp}^{2}$ law of the non-interacting case [20]. However, the intrachain interaction has drastic effects. Firstly, the total sum rule is strongly reduced compared to $V=0$ (shown as a reference on Fig. 3). Secondly, it is found that $\pi\left\langle D_{y y}\right\rangle_{\Phi_{y}}$ and $I_{y y}$ behave differently.

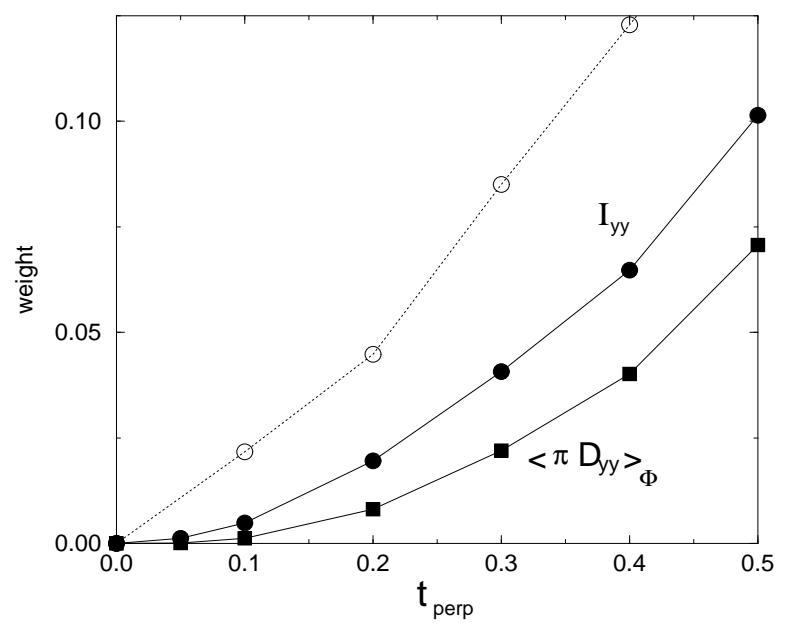

FIG. 3. $\pi\left\langle D_{y y}\right\rangle_{\Phi_{y}}(\boldsymbol{\square})$ and $I_{y y}(\bullet)$ as a function of $t_{\perp}$ for a $3 \times 8$ system and $V=2, i_{0}=3$. For comparison, the $V=0$ case is also displayed as a dotted line.

Let us consider the ratio $r=\pi\left\langle D_{y y}\right\rangle_{\Phi_{y}} / I_{y y}$ which corresponds to the relative part of the Drude weight in the total optical conductivity. In Fig. औ, we note that the ratio $r$ decreases as $t_{\perp}$ goes to 0 and can become rather small. 


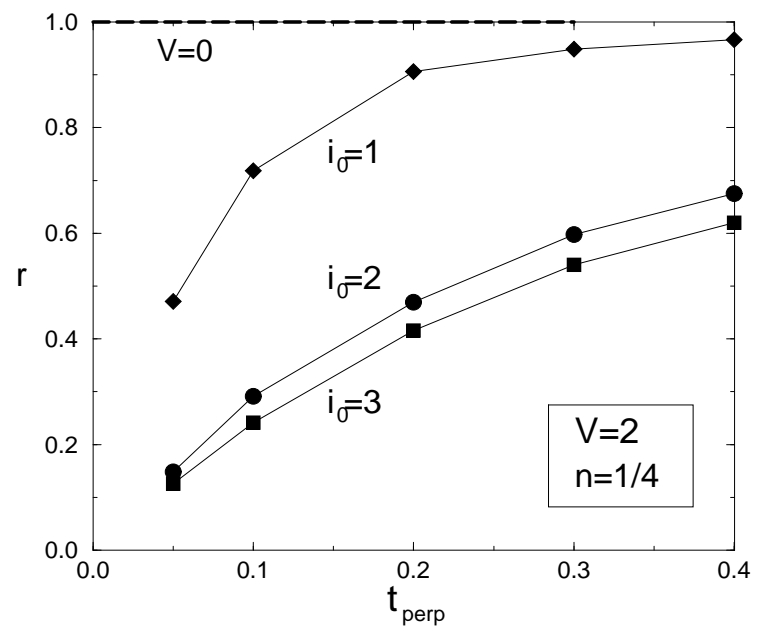

FIG. 4. Ratio of the Drude weight to the total conductivity in the $3 \times 8$ system as a function of $t_{\perp}$ for $\mathrm{NN}$ and longer range interaction with the same NN magnitudes $V=2$.

These results should have very important consequences on the experimental side. Indeed, our results predict, for small $t_{\perp}$, anomalous transport perpendicular to the chains even when $D_{y y}$ does not completely vanish (if $\left.\alpha<\alpha_{0}\right)$ since spectral weight is suppressed predominantly from the coherent part of the conductivity. In order to confirm this behaviour, the frequency dependent optical conductivity has been calculated directly by use of the Kubo formula,

$$
\sigma_{y y}^{r e g}(\omega)=\frac{\pi}{N} \sum_{n \neq 0} \frac{\left|\left\langle\phi_{0}\left|\hat{\jmath}_{y}\right| \phi_{n}\right\rangle\right|^{2}}{E_{n}-E_{0}} \delta\left(\omega-\left(E_{n}-E_{0}\right)\right)
$$

where $\hat{\mathrm{j}}_{y}$ is the transverse current operator and the sum runs over all the excited states.

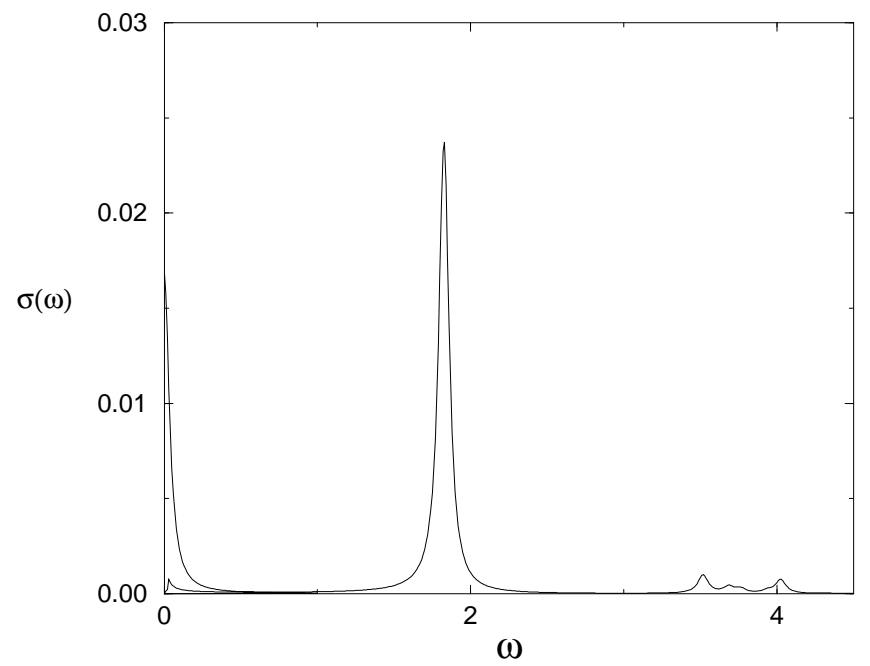

FIG. 5. Transverse optical conductivity vs frequency for a $3 \times 8$ system at quarter filling with $V=2, i_{0}=3$ and $t_{\perp}=0.1$. The Drude $\delta$-function has been represented with the same small imaginary part $\varepsilon=0.04$.
In the free case, the current operator commutes with the Hamiltonian and therefore, the conductivity only contains a Drude peak. However, as can be seen on Fig. 5 , for finite interaction strengths and in the $3 \times 8$ cluster, a pronounced structure appears at finite frequency. In order to determine more accurately the position in energy of the weight, we have computed the first moment of the distribution,

$$
\langle\omega\rangle=\int_{0^{+}}^{\infty} \sigma_{y y}^{r e g}(\omega) \omega d \omega / \int_{0^{+}}^{\infty} \sigma_{y y}^{r e g}(\omega) d \omega
$$

which is expected to behave smoothly with the various parameters. We observe that, once $t_{\perp}$ is turned on between the chains, weight immediately appears predominantly at finite frequencies (see Fig. 6). This typical frequency increases with the strength of the interaction. This is clearly a signature of some form of incoherent perpendicular transport.

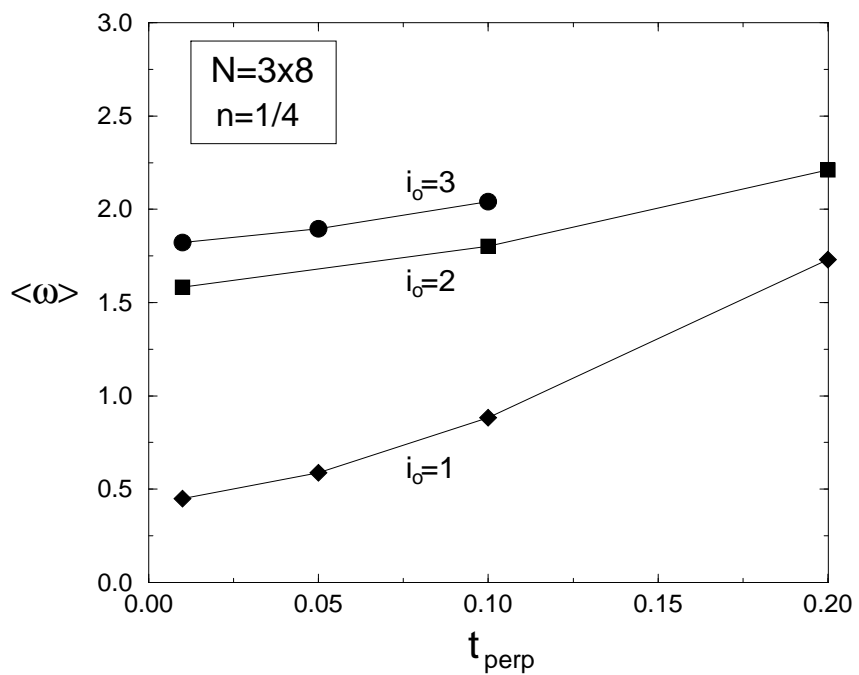

FIG. 6. First moment of the conductivity as a function of $t_{\perp}$ for $V=2$ and different interaction ranges.

In this work, different approaches to interchain coherence have been investigated. As a first step, we have focused on the energy splitting generated by the transverse hopping in the dispersion relation of the LL collective modes. By finite size scaling analysis, we have shown that this splitting was monitored by the LL parameter $\alpha$. However, incoherent interchain hopping is found for much smaller values of $\alpha$ than those predicted by the RG calculations [9]. Moreover, in the regime where $t_{\perp}$ is still relevant $\left(\alpha<\alpha_{0}\right)$, the most important results are that (i) the Drude weight and the total optical sum rule grow less rapidly with $t_{\perp}$ than in the non interacting case and (ii) even when the Drude weight remains finite (when $t_{\perp}$ is relevant), transverse transport is predominantly incoherent in the small $t_{\perp}$ regime. How small $t_{\perp}$ needs to be so that this regime is observed depends on the strength 
of the interaction. Typically, for $\alpha \sim 0.2$, strong suppression of coherent transport occurs up to $t_{\perp} \sim 0.15$. This phenomenon could explain the anomalous transport which is observed experimentally.

[1] S. Capponi, D. Poilblanc and F. Mila, Phys. Rev. B 54, 17547 (1996).

[2] P. W. Anderson, Phys. Rev. Lett. 67, 3844 (1991); P. W. Anderson, Science 256, 1526 (1992).

[3] S. L. Cooper and K. E. Gray in Physical Properties of High Temperature Superconductors IV, D. M. Ginsberg (ed.) (World Scientific), 1994; S. L. Cooper et al., Phys. Rev. Lett. 70, 1533 (1993); Y. Maeno et al., Nature 372, 532 (1994); X.-D. Xiang et al., Phys. Rev. Lett. 68, 530 (1992); K. Tamasaku, T. Ito, H. Takagi and S. Uchida, Phys. Rev. Lett. 72, 3088 (1994).

[4] D. G. Clarke, S. P. Strong, and P. W. Anderson, Phys. Rev. Lett. 74, 4499 (1995).

[5] F. D. M. Haldane, J. Phys. C 14, 2585 (1981).

[6] D. Jérome and H. Schulz, Adv. Phys. 31, 299 (1982).

[7] P. Wzietek, F. Creuzet, C. Bourbonnais, D. Jérome, K. Bechgaard and P. Batail, J. Phys. (Paris) I 3, 171 (1993).

[8] K. Behnia et al, Phys. Rev. Lett. 74, 5272 (1995).

[9] See D. Boies, C. Bourbonnais, and A.-M.S. Tremblay, Phys. Rev. Lett. 74, 968 (1995) and references therein.

[10] D. G. Clarke, S. P. Strong, and P. W. Anderson, Phys. Rev. Lett. 72, 3218 (1994).

[11] F. Mila and D. Poilblanc, Phys. Rev. Lett. 76, 287 (1996). (1993).

[12] C. Lanczos, J. Res. Natl. Bur. Stand. 45, 255 (1950).

[13] D. Poilblanc, H. Endres, F. Mila, M. Zacher, S. Capponi and W. Hanke, Phys. Rev. B 54, 10261 (1996).

[14] W. Kohn, Phys. Rev. 133, A 171 (1964).

[15] D. Poilblanc, Phys. Rev. B 44, 9562 (1991).

[16] C. Gros, Z. Phys. B 86, 359 (1992).

[17] J. Voit, Rep. Prog. Phys. 58, 977 (1995) and references therein.

[18] H. J. Schulz, "Correlated Electron Systems", p. 199, ed. V. J. Emery (World scientific, Singapore, 1993); H. J. Schulz, "Strongly Correlated Electronic Materials: The Los Alamos Symposium - 1993", p. 187, ed. K. S. Bedell, Z. Wang, D. E. Meltzer, A. V. Balatsky, E. Abrahams (Addison-Wesley, Reading, Massachussets, 1994).

[19] P. Maldague, Phys. Rev. B 16, 2437 (1977).

[20] For a finite system of non-interacting fermions, the $D_{y y}\left(t_{\perp}\right)$ curve is made of linear segments the slope of which increases with $t_{\perp}$. The $t_{\perp}^{2}$-law is recovered in the thermodynamic limit when the average length of the segments vanishes. This peculiar behaviour makes finite size scaling analysis of the transverse conductivity at finite $V$ difficult. 